Gynäk. Rdsch. 1970;9:233

\title{
Kann die Ultraschallüberwachung den Fetus schädigen?
}

Die optimale Uberwachung des Fetus in der Risikoschwangerschaft und des Nasziturus während der Geburt erfordert sorgfältige Kontrolle der Herzaktion. Die bisher gebräuchlichen Registriergeräte nehmen die Herztöne mittels eines Mikrofons auf. Eine bessere Registrierung ermög-licht die Ultraschallaufnahme aufgrund des Doppler-Prinzips, wie Be-richte von L. R. Bernstine (Bethesda, Md.), von E. H. Bishop (Philadelphia, Pa.) und von B. Sundén (Lund) u. a. ergeben. In Deutschland hat K. H. Mosler sich um die Einführung der kontinuierlichen Uberwachung der fetalen Herzaktionen vor und während der Geburt und wich-tige Verbesserungen dieser Methode verdient gemacht.1

Die Voraussetzung für die allgemeine Anwendung ist die Unschäd-lichkeit für den Fetus. Neuerdings kamen hier Zweifel auf durch Berichte über Chromoso-menschäden, die an Gewebskulturen bei experimenteller Untersuchung beobachtet wurden. Die grosse Bedeutung der Herztonüberwachung in der klinischen Geburtshilfe erfordert baldige Klärung. Zur ausführlichen Information der Gynäkologen haben wir die ungekürzte Ubersetzung einer vorläufigen Mitteilung von I. J. C. Macintosh und D. A. Davey über ihre experimentellen Untersuchungen sowie die Meinung mehrerer Experten hierzu zusammengestellt. H. Schwalm 1 K. H. Mosler: Dauerüberwachung der fetalen Herzaktionen unter der Geburt mittels Ultraschall. Experientia 25: 222 (1969). 